\title{
Orbital complications of acute rhinosinusitis in children: a retrospective review of 33 patients
}

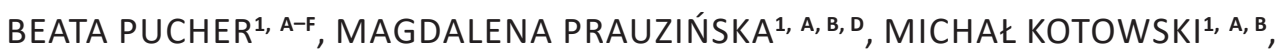 \\ AGATA KAŁUŻNA-M $Ł Y N A R C Z Y K^{1,8, F}$, PAULINA ADAMCZYK ${ }^{1,8, ~}$, \\ KATARZYNA JOŃCZYK-POTOCZNA ${ }^{2,}$ A, B, D, JAROSŁAW SZYDŁOWSKI ${ }^{1,}$ A, D \\ ${ }^{1}$ Pediatric Otolaryngology Department, Poznan University of Medical Sciences, Poland \\ ${ }^{2}$ Department of Pediatric Radiology, Poznan University of Medical Sciences, Poland
}

A - Study Design, B - Data Collection, C - Statistical Analysis, D - Data Interpretation, E - Manuscript Preparation, F - Literature Search, G - Funds Collection

Summary Background. Acute rhinosinusitis occurs commonly in children. Orbital complications are reported in $5 \%$ to $7 \%$ of patients. Orbital involvement is reported to occur more often in children than in adults.

Objectives. To evaluate the prevalence of orbital complications in children with acute rhinosinusitis.

Material and methods. A retrospective study of 33 children referred to our department with orbital complications of acute rhinosinusitis between January 2016 and March 2018 was carried out. Patients' charts were investigated for the following data: gender and morbidity rate in the different age groups. Diagnostic imaging - CT scans and/or X-ray of the paranasal sinuses were analyzed. The incidence of the stages of orbital complications and their primary treatment (conservative or surgery) was evaluated.

Results. Group 1 (0-3 years of age) included 7 children. Group 2 (children aged 4-10 ( $n=22)$ were affected most often. Group 3 (11-18 years of age) involved 4 patients. In a majority of patients, a CT scan was performed. An analysis of the stages of disease according to Chandler's classification revealed that stage I of orbital complications occurred most frequently, with a predominance in younger children.

Conclusions. The proper distinction between preseptal and orbital cellulitis, and subperiosteal abscess, is crucial in choosing the appropriate treatment strategy. Any delay may result in threatening vision complications.

Key words: sinusitis, orbital, child.

Pucher B, Prauzińska M, Kotowski M, Kałużna-Młynarczyk A, Adamczyk P, Jończyk-Potoczna K, Szydłowski J. Orbital complications of acute rhinosinusitis in children: a retrospective review of 33 patients. Fam Med Prim Care Rev 2018; 20(3): 259-262, doi: https://doi. org/10.5114/fmpcr.2018.78270.

\section{Background}

Acute rhinosinusitis occurs commonly in children. Orbital complications are reported in $5 \%$ to $7 \%$ of patients. Orbital involvement is reported to occur more often in children than in adults. Ethmoid sinus is the most commonly reported sinus to be involved in children. This is attributed to the close relation between the orbit and ethmoidal sinus in children, with a thin line separating them. In adults, the development of the frontal sinus makes it a frequent cause of orbital involvement, along with maxillary sinus. Sphenoid sinus involvement is rare and may lead to optic nerve involvement [1, 2].

Sinus infection can spread to involve orbital and intracranial structures directly, by retrograde extension or, rarely, by hematogenous spread. The orbit is separated from the ethmoid cells and the maxillary sinus by thin bony plates, which have naturally congenital bony dehiscence [3]. The infection can also be secondary to spread through the valveless ophthalmic veins with retrograde flow, complicating venous drainage, which provide direct pathways from the paranasal sinuses to the orbit (most commonly ethmoid sinusitis), leading to a progression of phlebitis [4].

In 1970, Chandler et al. proposed a classification that places patients into five groups: grade I - inflammatory edema (preseptal cellulitis), grade II - orbital cellulitis, grade III - subperiosteal abscess, grade IV - orbital abscess, grade V - cavernous sinus thrombosis. In all classifications proposed, proptosis seems to be the finding that differentiates the most severe stages of the complication. Proptosis indicates the presence of postseptal sinusitis complication. Proptosis may also be used as a guide to help locate the abscess. If the proptosis is symmetrical, there is an even greater chance of orbit content involvement. However, if asymmetrical, the abscess may be located in the opposite orbitary quadrant. In general, the larger the proptosis, the more severe the inflammation or the abscess size is $[2,3]$.

In patients who develop orbital cellulitis, $10 \%$ have temporary visual loss in the affected eye. Other complications may occur less frequently and include meningitis, frontal osteomyelitis, intracranial abscess, etc. The treatment of these complications requires a team of different specialists: a pediatric ENT specialist taking care of the sinus infection, an ophthalmologist evaluating the visual complications, a pediatrician taking care of the clinical problems. Differential diagnosis of orbital swelling should include facial infections, trauma, iatrogenic causes, tumors and dacryocystitis. However, in the pediatric population, sinusitis is responsible for a least $75 \%$ of cases, and orbital complication may be the first and only present sign of sinusitis [3].

\section{Objectives}

The aim of the study was to present the most common orbital complications of acute rhinosinusitis in children.

\section{Material and methods}

A retrospective study of 33 children referred to our department with orbital complications of acute rhinosinusitis between January 
2016 and March 2018 was carried out. The patients' charts were investigated for the following data: gender and morbidity rate in the different age groups (group 1-aged 0-3 years, group 2-aged 4-10 years, group 3 - aged 11-18 years). The ENT and ophthalmologic examination at admission (extraocular muscle motion, examination of periorbital adnexa and, when possible, visual acuity) were estimated in every case. The evaluated laboratory tests included white blood cell counts in the peripheral blood and level of CRP in blood serum. The results of swabs for microbiologic examinations taken either from the mucopus in the middle nasal meatus or from the pus obtained during drainage of subperiostal abscesses were also evaluated. Diagnostic imaging - CT (computed tomography) scans and/or X-ray of the paranasal sinuses - were analyzed. The incidence of the stages of orbital complications (according to Chandler's classification) and their primary treatment (conservative or surgery) was evaluated. The treatment protocol included intravenous broad-spectrum antibiotics, nasal decongestants and analgesics for patients with stage I and II of the disease. When no improvement was observed within the first 48 hours of treatment in stage $\mathrm{II}$, endoscopic surgery preceded by CT imaging with contrast was performed. In stage III, surgical treatment was introduced within 24 hours of admission and included endoscopic surgery with abscess drainage. Antibiotic therapy was initiated for 10 to 14 days. In complicated acute rhinosinusitis, high-dose amoxicillin-clavulanate or cephalosporins are recommended. If they fail, ceftriaxone or cefotaxime are introduced. Adjunctive therapy also included saline nasal irrigation, systemic decongestants after surgical intervention and intranasal corticosteroids.

The paper presents a retrospective study, which does not require an opinion from the Ethics Committee.

\section{Results}

A total number of 33 patients were enrolled in the study (20 boys $-60.6 \%$ and 13 girls $-39.4 \%$ ). Children aged $4-10$ were affected most often ( $n=22,66.7 \%)$. Group 1 ( $0-3$ years of age) included 7 children (21.2\%), and group 3 (11-18 years of age) involved 4 patients $(12.1 \%)$

According to patients' history, orbital symptoms appeared after rhinitis a few days prior. There was no history of midface trauma, dental infection or conjunctivitis reported. Anterior rhinoscopy revealed the presence of mucopus in the middle nasal meatus in 26 children, and erythematous and congested turbinates in 7 patients. In children with preseptal cellulitis $(n=17)$, symptoms included unilateral $(n=11)$ or bilateral $(n=6)$ eyelid swelling, erythema and tenderness (Figure 1). Visual acuity, pupillary reaction and extraocular

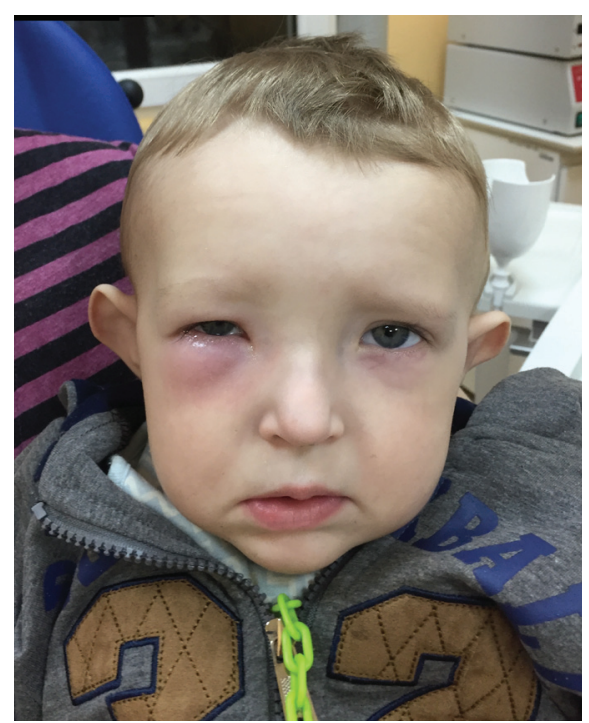

Figure 1. Preseptal cellulitis in 5-year-old boy (caregivers' written consent was obtained to publish the patient's photo)

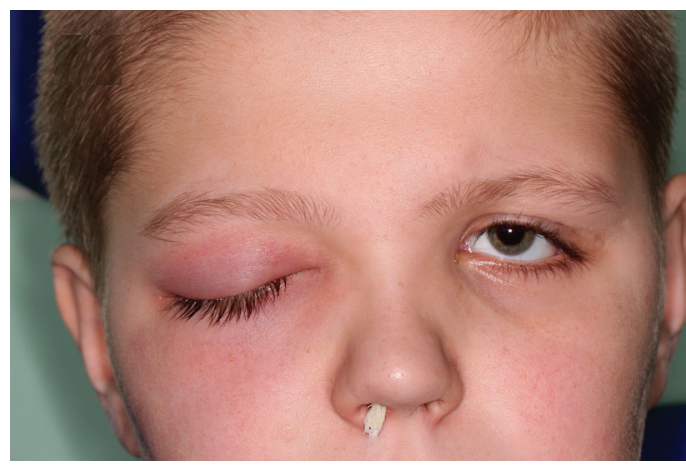

Figure 2. Eyelid swelling and ophtalmophlegia of the right eye in 6-year-old boy (caregivers' written consent was obtained to publish the patient's photo)

motility were normal. In children with stage II of the disease $(n=9)$, eyelid edema, erythema, mild proptosis and chemosis were present (Figure 2). Visual acuity, according to an ophthalmologist's examination, was not impaired. In cases with subperiosteal abscess (III stage of the disease, $n=7$ ), the examination revealed unilateral eyelid edema, erythema, as well as larger proptosis and gaze restriction. There was no deterioration of visual acuity.

In laboratory findings, there was elevated CRP and leucocytosis in all children. Microbiology results (obtained in 30 patients) presented that Streptococcus pneumoniae was the most frequent bacteria cultured $(n=10)$, followed by Haemophilus influenza ( $n=$ 4), Staphylococcus aureus $(n=4)$ and Corynebacteriae $(n=1)$. In 4 children, polymicrobial cultures were identified, and in 7 , the result was negative.

In a majority of patients, a CT was performed ( $n=24,72.7 \%)$, in $2(6.1 \%)$ - an X-ray of the paranasal sinuses was made before referral to our department. A total of 7 (21.2\%) received no imaging, as they had only eyelid swelling without other symptoms, which rapidly responded to antibiotic therapy. Every patient with whom surgery was necessary received CT imaging beforehand. The most common sinus involved was the ethmoid $(n=26$, with radiologic imaging), and in 15 children, both the ethmoid and the maxillary sinus were involved. Analysis of the disease stages according to Chandler's classification revealed that stage I orbital complications occurred most frequently ( $n=17,51.58 \%)$, with a predominance in younger children (Table 1).

\begin{tabular}{|c|c|c|c|c|}
\hline $\begin{array}{l}\text { Stage of the disease } \\
\text { (according to Chan- } \\
\text { dler's classification) }\end{array}$ & $\begin{array}{l}\text { Group } 1 \\
(n=7)\end{array}$ & $\begin{array}{l}\text { Group 2 } \\
(n=22)\end{array}$ & $\begin{array}{l}\text { Group } 3 \\
(n=4)\end{array}$ & $\begin{array}{l}\text { Total } \\
(n=33)\end{array}$ \\
\hline I: preseptal cellulitis & \begin{tabular}{|l}
5 \\
$(71.4 \%)$
\end{tabular} & \begin{tabular}{|l}
12 \\
$(54.5 \%)$
\end{tabular} & $\begin{array}{l}0 \\
(0.00)\end{array}$ & \begin{tabular}{|l}
17 \\
$(51.5 \%)$
\end{tabular} \\
\hline II: orbital cellulitis & $2(28.6 \%)$ & $4(18.2 \%)$ & $3(75 \%)$ & 9 (27.3\%) \\
\hline $\begin{array}{l}\text { III: subperiosteal } \\
\text { abscess }\end{array}$ & $\begin{array}{l}0 \\
(0.00)\end{array}$ & \begin{tabular}{|l}
6 \\
$(27.3 \%)$
\end{tabular} & $\begin{array}{l}1 \\
(25 \%)\end{array}$ & \begin{tabular}{|l}
7 \\
$(21.2 \%)$
\end{tabular} \\
\hline IV: orbital abscess & $0(0.00)$ & $0(0.00)$ & $0(0.00)$ & $0(0.00)$ \\
\hline $\begin{array}{l}\text { V: cavernous sinus } \\
\text { thrombosis }\end{array}$ & $0(0.00)$ & $0(0.00)$ & $0(0.00)$ & $0(0.00)$ \\
\hline
\end{tabular}

A total of 17 patients (stage I) received conservative treatment, with the need of an antral puncture in 2 due to the fluid level in the maxillary sinus. In 5 children (out of 9 with stage II), surgical treatment was necessary, as they did not respond to conservative treatment. In 7 patients with stage III disease, surgical intervention was required within 24 hours after admission and was preceded by $\mathrm{CT}$ imaging, which revealed the presence of a subperiosteal abscess (Figure 3). The surgical procedure entailed functional endoscopic sinus surgery (FESS), which was performed in 5 children with stage II of the disease, and FESS with endoscopic subperiosteal abscess 
drainage was undertaken in 7 patients with stage III. The postoperative course was not remarkable, and the mean hospital stay was 7.1 days (min. 1 day, max. 13 days). All children were followed-up in the outpatient clinic 2 and 6 weeks after discharge - there was no record of recurrence of the disease.

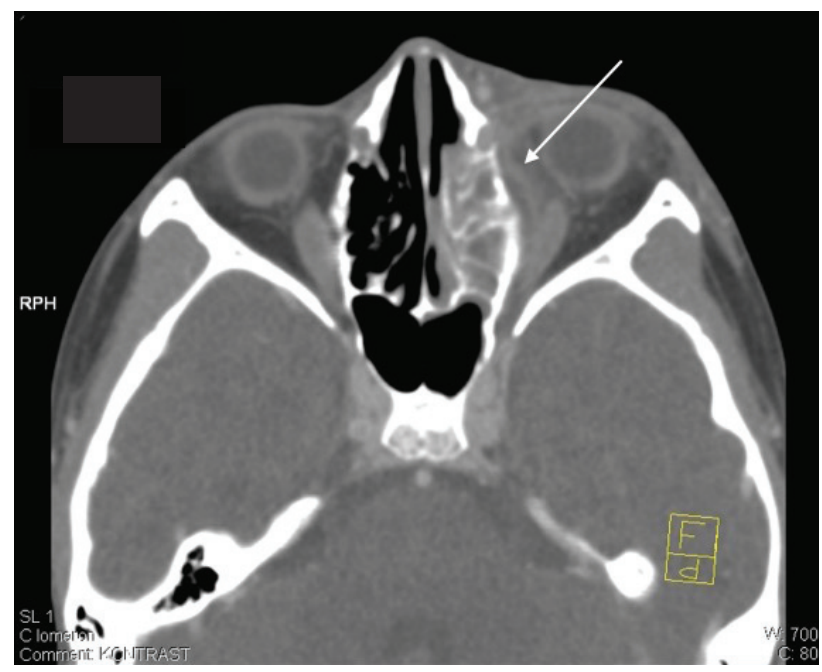

Figure 3. CT scan of subperiosteal abscess of the right orbit (indicated with an arrow)

\section{Discussion}

Orbital complications are observed more often in younger children, while intracranial complications typically occur in older children. The ethmoid sinuses are the most common source of infection in small children [4-9]. According to Watts, orbital cellulitis, secondary to sinusitis, is almost exclusively due to ethmoidal sinusitis in the first 5 years of life and is secondary to ethmoidal, maxillary and frontal sinusitis in children over 7 years of age [8].

According to Brook, the most common pathogens in cellulitis and abscesses are those seen in acute and chronic sinusitis, depending on the length and etiology of the infection. These are: S. pneumoniae, $\mathrm{H}$. influenzae, Staphylococcus aureus and anaerobic bacteria [3]. A microbiological study was performed in our 30 cases of orbital complications, either by obtaining intraoperative samples of pus or with swabs of nasal secretions. Specific bacteria were identified in only 23 out of 30 cases, perhaps because some children received antibiotic therapy before referral to the ENT Dept.

The orbital complications include preseptal cellulitis, orbital cellulitis and abscess formation $[1,10]$. Preseptal cellulitis is an infection of an eyelid and adjacent skin anterior to the orbital septum. This was the most common orbital complication in our study, and it predominantly affected children under 10 years of age (Figure 3 ). Treatment includes broad-spectrum antibiotics, which may be oral in mild cases providing that the follow-up is ensured. Admission for intravenous antibiotics is usually recommended in children under 3 years of age [11]. Orbital cellulitis develops when the infection spreads posterior to the orbital septum. This condition can lead to an abscess if not treated properly. The main symptoms include eyelid edema and erythema, mild proptosis and chemosis. Motility may be limited, but visual acuity remains normal. These patients require hospitalization and intravenous antibiotic therapy and an ophthalmology examination. A CT of the sinuses and orbits is required [11]. The progress of ophthalmological signs, such as proptosis, conjunctival injection and limitation of extraocular movements, is highly suggestive of an intraorbital process and requires radiological examination [10]. The investigation of choice is a contrastenhanced sinus $C T$ in order to verify the diagnosis and to assess the severity of the subperiosteal abscess $[1,6,11]$. Indications are a ring-enhanced lesion or presence of air-fluid level in the extraocular space, displacement and enlargement of the medial rectus muscle and proptosis. Treatment consists of immediate administration of intravenous antibiotics and surgical treatment, which includes transnasal endoscopy with ethmoidectomy, skeletonizing and penetrating the lamina papyracea in order to perform drainage, as was performed in 7 of our cases of a subperiosteal abscess [11]. Transnasal and external approaches are occassionally required for better exposure.

In stage IV of the disease, the infection exceeds the orbital periosteum, and an orbital abscess is formed. Symptoms include evident proptosis, chemosis, complete ophthalmoplegia and visual impairment. An orbital abscess is a risk for progression to irreversible blindness, and surgical intervention is mandatory. In stage $\mathrm{V}$ - the infection from the sinuses and the orbit can spread to the cavernous sinus through the free anastomosis, valveless vein system and the superior and inferior ophthalmic veins, causing cavernous sinus thrombosis. A sign of stage $\mathrm{V}$ of the disease is a progression of symptoms to the opposite eye. A physical examination reveals the rapid progress of chemosis and ophthalmoplegia. The patient's condition may worsen to loss of vision, meningitis and death [11]. Imaging studies include a CT scan and MRI with contrast. Treatment requires high doses of intravenous antibiotics that cross the blood-brain barrier for 6-8 weeks and surgical drainage of the affected sinuses. In our material, there were no cases with stage IV and V of the disease. Intracranial complications are less frequent than orbital and are seen more often in frontal or sphenoid sinusitis [11].

\section{Conclusions}

The most common orbital complication of acute rhinosinusitis in children is preseptal cellulitis. The ethmoid sinus is the main source of the complication, with a predominance in younger children.

The proper distinction between preseptal and orbital cellulitis, and subperiosteal abscess, is crucial in choosing the appropriate treatment strategy. Preseptal cellulitis responds to conservative treatment. An orbital abscess requires immediate surgical intervention involving decompression of the orbit, abscess drainage and opening of the affected sinuses $[1,10]$.

Source of funding: This work was funded by the authors' own resources. Conflict of interest: The authors declare no conflict of interests.

\section{References}

1. Al-Madani MV, Ahmed Essa Khatatbeh AE, Rawashdeh RZ, et al. The prevalence of orbital complications among children and adults with acute rhinosinusitis. Braz J Otorhinolaryngol 2013; 79(6): 716-719.

2. Welkoborsky HJ, Graß S, Deichmüller C, et al. Orbital complications in children: differential diagnosis of a challenging disease. Eur Arch Otorhinolaryngol 2015; 272: 1157-1163.

3. Brook I. Microbiology and antimicrobial treatment of orbital and intracranial complications of sinusitis in children and their management. Int J Ped Otorhinolaryngol 2009; 73: 1183-1186.

4. Buchanan MA, Muen W, Heinz P. Management of periorbital and orbital cellulitis. Pediatr Child Health 2012; 22(2): 72-77.

5. Cohen SM. Orbital cellulitis as a complication of sinusitis. J Nurse Pract 2011; 7(1): 38-44.

6. Ibrahim AA, Hussein WKA, Omran AA. Post-septal orbital complications of acute bacterial rhinosinusitis: endoscopic anatomical considerations. Egypt J Ear Nose Throat and Allied Sci 2015; 16: 209-215. 
7. Sharma PK, Saikia B, Sharma R. Orbitocranial complications of acute sinusitis in children. J Emerg Med 2014; 47(3): $282-285$.

8. Watts P. Preseptal and orbital cellulitis in children: a review. Pediatr Child Health 2011; 22(1): 1-8.

9. Lee S, Yen MT. Management of preseptal and orbital cellulitis. Saudi J Ophthalmol 2011; 25(1): 21-29.

10. Teinzer F, Stammberger H, Tomazic PT. Transnasal endoscopic treatment of orbital complications of acute sinusitis: the Graz concept. Ann Otol Rhinol Laryngol 2015; 124(5): 368-373.

11. Ebner Y, April MM. Pediatric sinusitis. In: Licameli GR, Tunkel DE, eds. Pediatric otorhinolaryngology: diagnosis and treatment. Noida, Uttar Pradesh: Thieme; 2013: 66-74.

Tables: 1

Figures: 3

References: 11

Received: 15.05.2018

Reviewed: 04.06.2018

Accepted: 08.07.2018

Address for correspondence:

Beata Pucher, MD, PhD

Klinika Otolaryngologii Dziecięcej

Katedra Otolaryngologii i Onkologii Laryngologicznej UM

ul. Szpitalna $27 / 33$

60-572 Poznań

Polska

Tel.: +48 618 491-363

E-mail: bpucher@poczta.onet.pl 